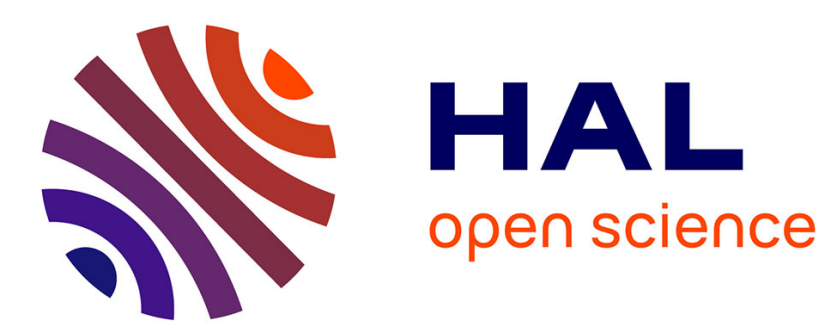

\title{
A numerical model for multidirectional wear
}

Franck Jourdan

\section{To cite this version:}

Franck Jourdan. A numerical model for multidirectional wear. Wear, 2010, 268 (11-12), pp.1380-1386.

10.1016/j.wear.2010.02.012 . hal-00686628

\section{HAL Id: hal-00686628 \\ https://hal.science/hal-00686628}

Submitted on 10 Apr 2012

HAL is a multi-disciplinary open access archive for the deposit and dissemination of scientific research documents, whether they are published or not. The documents may come from teaching and research institutions in France or abroad, or from public or private research centers.
L'archive ouverte pluridisciplinaire HAL, est destinée au dépôt et à la diffusion de documents scientifiques de niveau recherche, publiés ou non, émanant des établissements d'enseignement et de recherche français ou étrangers, des laboratoires publics ou privés. 


\title{
A numerical model for multi-directional wear
}

\author{
Franck Jourdan \\ Laboratoire de Mécanique et Génie Civil, Université Montpellier 2/CNRS UMR 5508, \\ Place Eugène Bataillon, CC 048, F-34095 Montpellier Cedex 5, France
}

\begin{abstract}
Many work showed that the change of sliding direction is a factor worsening wear between two bodies in contact. The objective of this study is to propose a model of wear able to reply these observations. This model tries to show that part of the multidirectional effects is due to the variation of wear rate according to the number of cycles. The results presented in this paper come from numerical calculations. They show that using a wear coefficient depending on the sliding distance, one can obtain, for a rectangular motion pattern, a volumetric wear up to 2 times more important than for a rectilinear motion pattern.
\end{abstract}

Key words: Multidirectional wear, Numerical method, Finite Elements, Nonlinear wear rate, Modelisation

\section{Introduction}

The works of Wang [1], Laurent et al.[2] and Turell et al.[3] clearly show the multidirectional effects of wear by comparing rectilinear motion pattern with rectangular motion pattern. They underline a significant increase in volumetric wear when the friction path changes of direction. For the same total distance from friction and the same load, volumetric wear can be up to 10 times greater depending on the form of the motion pattern. These observations are essential to a better understanding of the behaviour of wear. They suggest the need for particular care in the experimental and numerical modeling of wear mechanisms. To explain these multidirectional phenomena, Laurent et al.[2] propose an approach based on the dependence of wear on the sliding angle, whereas Turell et al.[3] propose a model of wear depending on the dimensions of the motion pattern. The physics of these multidirectional 
effects is likely to be varied in nature. It depends on the couple of materials in contact, their surface quality, roughness, thermomechanical properties, etc. This paper, proposes to allot part of this volumetric wear increase to the dependence of wear rate on the number of cycles and more precisely to the sliding distance. Indeed, many studies on wear [4], [5], [6], [7], show that wear rate drops when the number of cycles increases. The results show that the abrasive potential of the bodies in contact gradually decreases with repeated passages. Part of the multidirectional effects of wear are accounted for by using the preceding observation. To do so, a numerical model of wear is presented, based on previous works [8], [9] and [10], which was adapted to take into account a wear rate depending on the sliding distance. The dependence of wear rate is calculated by a variation of the wear coefficient. The numerical results, obtained with this new model of wear, are then presented. The numerical tests reproduce the experimental protocol suggested by Turell et al.[3].

\section{Preliminary observations}

This paragraph refers to the context of the study to reveal the points used to construct the model of wear. The first point raised relates to the experiments performed in Turell et al.[3] on multidirectional effects. The experimental protocol and results are presented, followed by a geometrical analysis of the zones of friction. The second point is based on experimental results to show that wear rate drops as the number of cycles increases.

\subsection{Multidirectional effects}

First, the experimental protocol was chosen which allowed Turell et al.[3] to highlight the multidirectional effects on the behavior of wear. Their work results primarily from biomechanics investigations and validates a theory suggested 3 years earlier by Wang[1]. The material couple used was composed of Ultra-High Molecular Weight PolyEthylene (UHMWPE) and of ChromiumCobalt (Co-Cr). The tests were carried out on a multidirectional machine of OrthoPOD type. The bodies in polyethylene were $9 \mathrm{~mm}$ diameter cylindrical pin. Six different rectangular motion patterns were tested. Curves of figure 1, after Turell et al.[3], represent, for each motion pattern, volumetric wear

according to the number of cycle. One notes a significant difference between the various tests. 


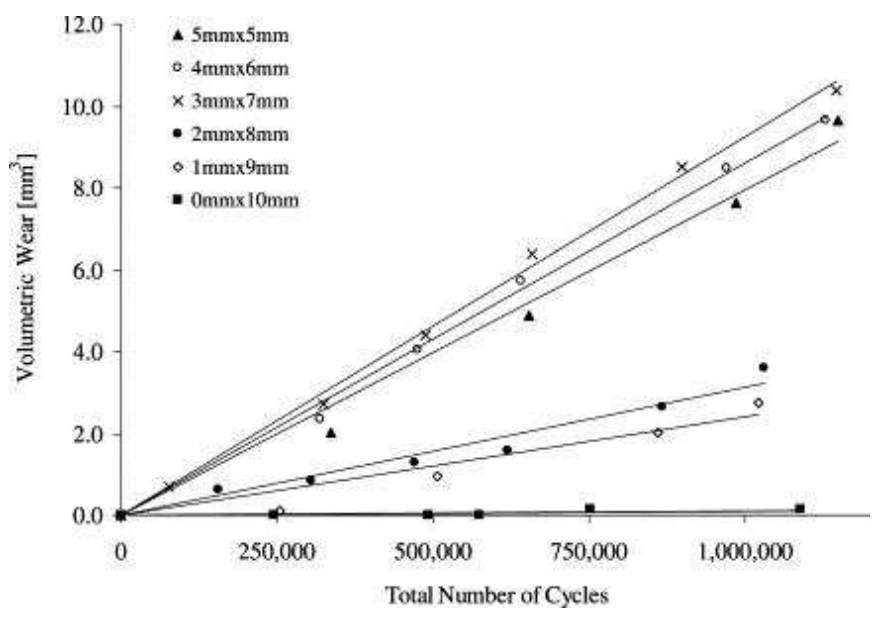

Figure 1: Comparison of volumetric wear rate for five different rectangular motion path patterns and linear tracking. Turell et al.[3]

Now let us look more closely at the surface of wear on the plate of Co-Cr. In the diagram of figure 2 , the discs represent the surface in contact under the UHMWPE pin at various steps of the cycle for a rectangular motion pattern. Numbers located in the various zones of figure 2 represent the number of times that these zones are rubbed during a cycle. It is noted that for one cycle, there are zones less rubbed than others. The dimensions of these zones vary with the geometrical characteristics of the rectangular motion pattern.

\subsection{Nonlinear wear rate}

For all materials in contact, one can note that the wear rate decreased when the number of cycles increased. This is supported by the works of [4], [5], [6], and [7]. A representative result is that obtained by Shi et al [4]. The figure 3 represents UHMWPE weight loss according to the number of cycles. It is noted clearly that, for this material couple, the wear rate drops when the number of cycles increases. This means that the abrasive properties of the rubbed zone decrease when the number of cycles increases. This decrease may come from a smoothing of counterface roughness. This is what is observed in figure 10 of paper of Samyn et al [11], after large-scale sliding.

According to the purely geometrical observations of figure 2 and knowing that the abrasive properties of the rubbed zone decrease when the number of cycles increases, it will be shown that part of the multidirectional effect is 


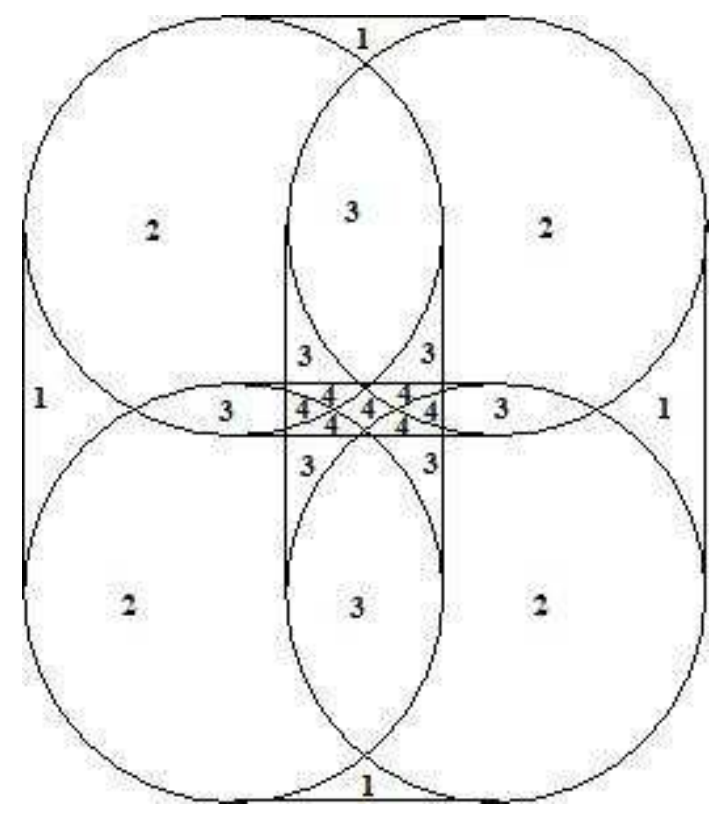

Figure 2: Contact surfaces at various stages of the cycle for a rectangular motion pattern

due to the variation of wear rate according to the cumulative sliding distance. Indeed, for rectangular motion patterns the total rubbed surface is much greater than for a rectilinear motion pattern and this increase in rubbed surface corresponds to less rubbed zones that are therefore more abrasive. Wear must thus be greater. This assumption now needs to be checked and the increase must be quantified. This is the subject of the following paragraphs.

\section{Law of wear}

Archard's law [12] proposes a linearization of the experimental results presented in the preceding paragraph(figure 3 ), in the following form:

$$
W=K s F
$$

where $W$ is the volume of wear, $K$ is the global coefficient of wear between the two bodies, $s$ the sliding distance and $F$ the applied load. While insisting on the nonlinearity of the results and with the aim of explaining directional effects, a nonlinear law of wear is proposed, of the form:

$$
W=K(s) F
$$




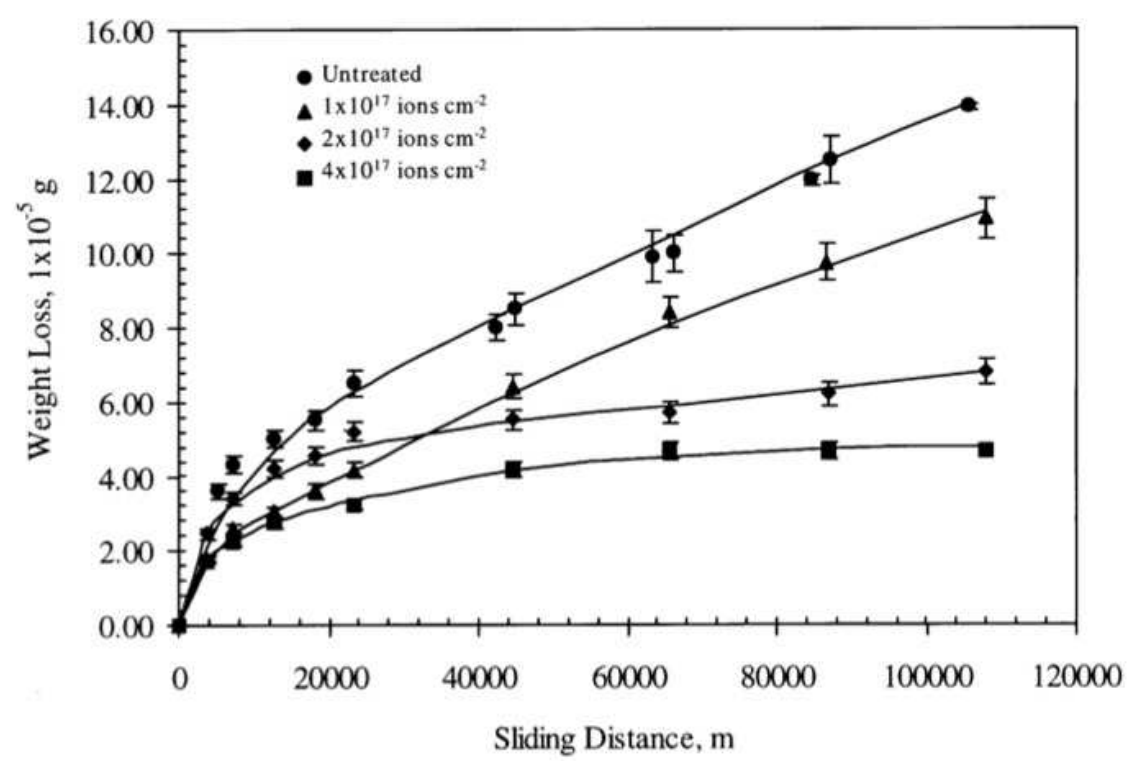

Figure 3: Variation of the UHMWPE weight loss as a function of the number of cycles and plasma immersion ion implantation. [4]

Given that the abrasive potential of materials in contact is a function of the cumulative sliding distance undergone by surface in contact, it is advisable to specify the variable $s$. To do so, the following observation is used. One considers a rectilinear form of slip between two bodies $S 1$, of lenght equal to $d_{1}$, and $S 2$, of lenght equal to $d_{2}$. (see figure 4 ).

For one cycle, each particle of $S 1$ rubs over a distance equal to $2\left(d_{2}-d_{1}\right)$ whereas the particles of $S 2$ rub over a distance equal to a maximum of $2 d_{1}$. For $n$ cycles, one define $s 1=2 n\left(d_{2}-d_{1}\right), s 2=2 n d_{1}$ and the preceding nonlinear law is enriched by

$$
W=\left(K_{1}(s 2)+K_{2}(s 1)\right) F
$$

where $K_{1}$ is the coefficient of wear for material associated with body $S 1$ and $K_{2}$ that associated with $S 2$. Thus, the coefficient of wear $K$ is a combination of coefficients depending on the cumulative distances from opposite surfaces. The volume $K_{2}(s 1) F$ is that lost by body $S 1$, and $K_{1}(s 2) F$ is that lost by body $S 2$. The coefficients $K_{1}$ and $K_{2}$ can be given by measuring the volume of wear lost by each solid. This law of wear (1) allows a total volume of wear to be predicted, but does not allow the wear on each body to be located. To 


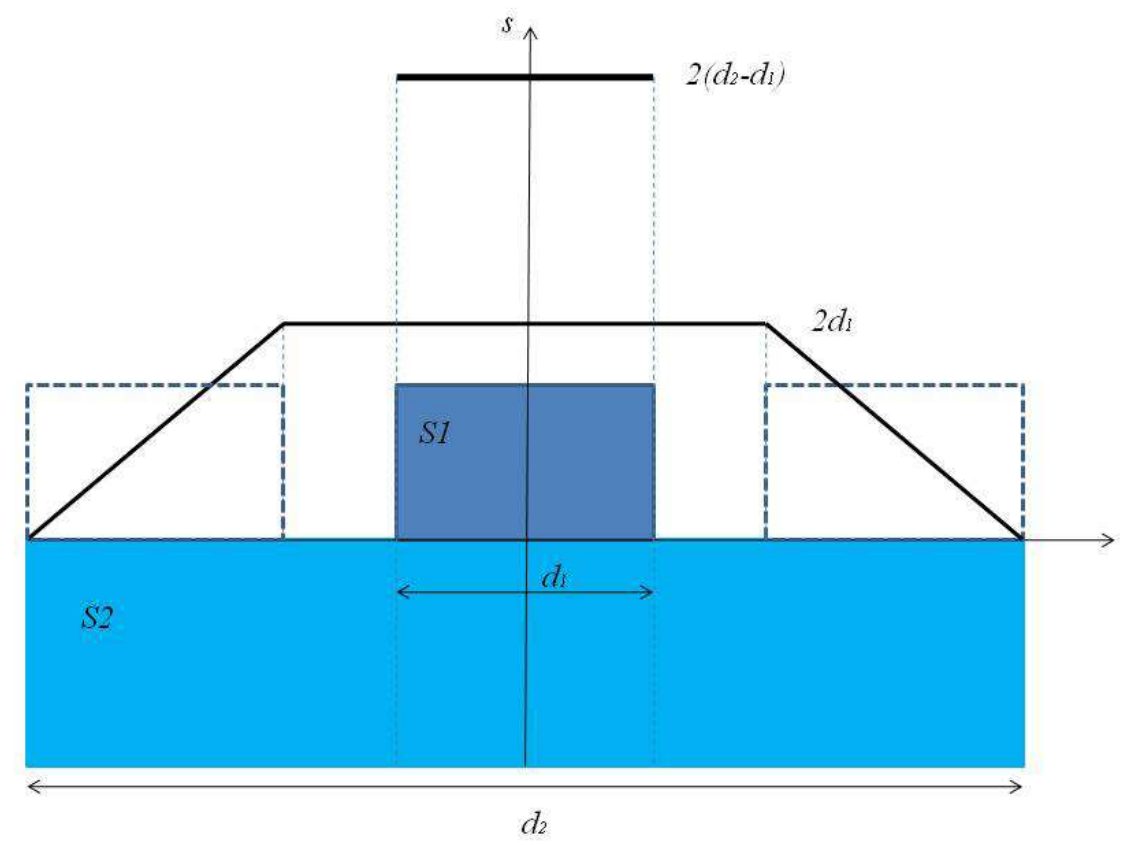

Figure 4: sliding distance

obtain this local information, a local model of wear is drawn from this law.

\section{Local wear model}

The model presented in this paragraph takes as a starting point the preceding works [9], [10] and the nonlinear law (1).

Let $\Omega_{O}$ be the domain at time $t=0$ and $\underline{\mathrm{X}}$ be the initial position of a particle of the body, then the transformation operator $\varphi$ gives the position $\underline{x}$ of the particle at time $t$, as follows

$$
\underline{x}=\underline{\varphi}(\underline{X}, t)
$$

The transformation operator $\underline{\varphi}$ is split in two parts.

$$
\underline{\varphi}(\underline{X}, t)=\underline{\varphi}^{e}(\underline{X}, t)+\underline{\varphi}^{w}(\underline{X}, t)
$$


where $\underline{\varphi}^{w}$ is transformation due to wear and $\underline{\varphi}^{e}$ is the complementary part (reduced in this study to the elastic contribution). Transformation $\underline{\varphi}^{w}$ is assumed to be nil beyond the contact zone. Before developing the evolution of transformation due to wear in the contact zone, it is necessary to introduce some notations relative to contact conditions. Two bodies are considered as contact candidates. Vectors $\underline{T}_{1}$ and $\underline{T}_{2}$ of the local frame $\left(\underline{T}_{1}, \underline{T}_{2}, \underline{N}\right)$ are chosen in the common tangent plane of the two bodies. Vector $\underline{N}$ is the normal vector at the tangent plane such that $\left(\underline{T}_{1}, \underline{T}_{2}, \underline{N}\right)$ is orthonormal (see Figure 5).

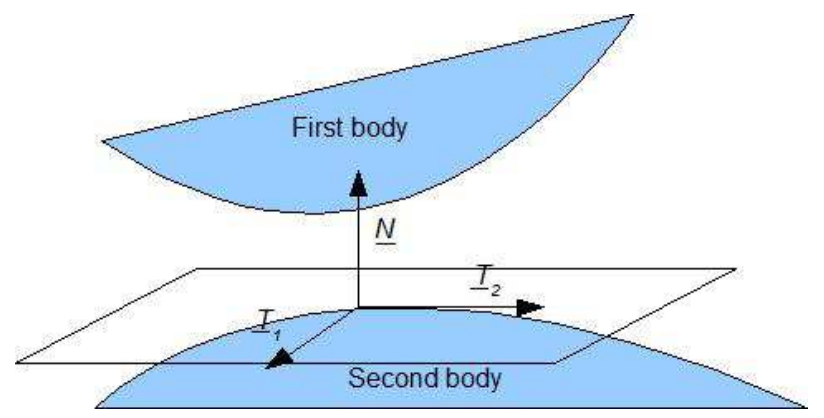

Figure 5: Local frame

Let $\underline{\varphi}_{1}$ be the transformation operator of the first body, and $\underline{\varphi}_{2}$ the transformation operator of the second body. The particle $P_{1}$ of body $S 1$, of initial position $\underline{X}_{1}$ and the particle $P_{2}$ of body $S 2$, of initial position $\underline{X}_{2}$ are in contact at time $t$ and then

$$
\underline{x}=\varphi_{1}\left(\underline{X}_{1}, t\right)=\varphi_{2}\left(\underline{X}_{2}, t\right)
$$

The relative velocity $\underline{v}$ is defined as

$$
\underline{v}=\underline{\dot{\varphi}}_{1}\left(\underline{X}_{1}, t\right)-\underline{\dot{\varphi}}_{2}\left(\underline{X}_{1}, t\right)
$$

and the tangential relative velocity $\underline{v}_{T}$ is the projection of $\underline{v}$ on the common tangent plane. Let $\underline{r}=\left(r_{T 1}, r_{T 2}, r_{N}\right)$ be the reaction force exerted on the first body, expressed in the local frame, so the normal component of velocity due to wear, is assumed to be governed by the following relationship :

$$
\left\{\begin{array}{l}
\frac{d}{d t}\left(\underline{\varphi}_{1}^{w} \cdot \underline{N}\right)=k_{1}^{w}\left(P_{1}, s_{2}\right) r_{N}\left|\underline{v}_{T}\right| \\
\frac{d}{d t}\left(\underline{\varphi}_{2}^{w} \cdot \underline{N}\right)=-k_{2}^{w}\left(P_{2}, s_{1}\right) r_{N}\left|\underline{v}_{T}\right|
\end{array}\right.
$$


The tangential component is assumed to be nil:

$$
\underline{\varphi}_{i}^{w} \cdot \underline{T}_{j}=0 \quad \forall i, j=1,2
$$

The local wear factor $k_{i}^{w}$ of the body $i=1,2$ is defined as

$$
k_{i}^{w}=\frac{1}{A\left(P_{i}\right)}\left(\frac{\partial K_{i}\left(s_{j}\right)}{\partial s_{j}}\right) \quad i \neq j
$$

where $A\left(P_{i}\right)$ is the elementary surface associated with the particle $P_{i}$ of position $\underline{x}$ at time $t$. Then, for particle $P_{i}$ of body $S i$, the local wear factor $k_{i}^{w}\left(P_{i}, s_{j}\right), \quad i \neq j$, depends on the cumulative distance $s_{j}$ of the opposite particle $P_{j}$ of body $S j$. This distance is defined as

$$
s_{j}(t)=\int_{0}^{t}\left|\underline{v}_{T}\left(\underline{\mathrm{X}}_{j}, \tau\right)\right| d \tau
$$

This wear law has to be considered in the context of frictional contact laws. Unilateral Signiorini's conditions and the Coulomb's dry friction law were chosen for this study. If $g_{N}=\left(\underline{\varphi}_{1}-\underline{\varphi}_{2}\right)$. $\underline{N}$ denotes the gap between the two bodies, unilateral Signiorini's conditions can be expressed in the following variational equation form

$$
r_{N}=\operatorname{proj}_{\Re^{+}}\left(r_{N}-\rho_{n} g_{N}\right)
$$

where $\operatorname{proj}_{\Re^{+}}$denotes the orthogonal projection on the positive real set $\Re^{+}$ and $\rho_{N}$ is a positive definite real number. Let $\underline{r}_{T}$ be the tangential part of the reaction force, then Coulomb's law is governed by the following equation

$$
\underline{r}_{T}=\operatorname{proj}_{C\left(r_{N}\right)}\left(\underline{r}_{T}-\rho_{T} \underline{v}_{T}\right)
$$

where $C\left(r_{N}\right)$ is the section of the Coulomb cone $\left\{\underline{r}_{T} \in \Re^{2}\right.$ such that $\left.\left\|\underline{r}_{T}\right\| \leq \mu r_{N}\right\}$, $\mu$ is the friction coefficient and $\rho_{T}$ is a positive definite real number. The parameters $\rho_{N}$ and $\rho_{T}$ are neither Lagrange multipliers, nor penalty coefficients. Their values are not necessarily large and do not have physical significances. However, for numerical reasons, they have optimal values.

\section{Numerical method}

The equations (5 - 9) are coupled via sliding velocities and contact reaction forces. The numerical resolution of this strongly coupled problem is 
rather technical. Thus, the reader is referred to the following articles [9], [10]. However, it should be specified that space discretization of the motion equations of bodies in contact is obtained using the finite element method and time discretization using a $\theta$-method. The frictional contact solver is based on the "Non Smooth Contact Dynamics" method proposed by M. Jean [13]. It was initially developed for rigid bodies and applied to deformable bodies in [14], [15]. As shown in [16], it is a non linear Gauss Seidel algorithm.

What is new in the present paper, is the taking into account of a nonlinear wear factor $k^{w}$.

Each body is meshed and every finite element of the contact surfaces represents a zone of friction. For each node $P_{i}$ of body $S i, i=1,2$, candidate to contact with body $S j, j \neq i$, its antagonist particle $P_{j}$ is introduced, located on body $S j$. The particle $P_{j}$ is the orthogonal projection of $P_{i}$ on $S j$ (see figure 6). This approach, assumes that each antagonist particle $P_{j}$ of the same element has the same cumulative sliding distance $s_{j}$ computed as follows:

$$
s_{j}(t+h)=s_{j}(t)+h \sum_{P_{i}}\left|\underline{v}_{T}\left(\underline{X}_{i}, t+h\right)\right|
$$

where $h$ is the time step and $\underline{X}_{i}$ is the initial position of $P_{i}$. The sum is for all nodes $P_{i}$ of $S i$ in contact with the friction zone during the time step. The cases where projection falls on an edge or a node were treated except for. An average value of the joint zones was used.

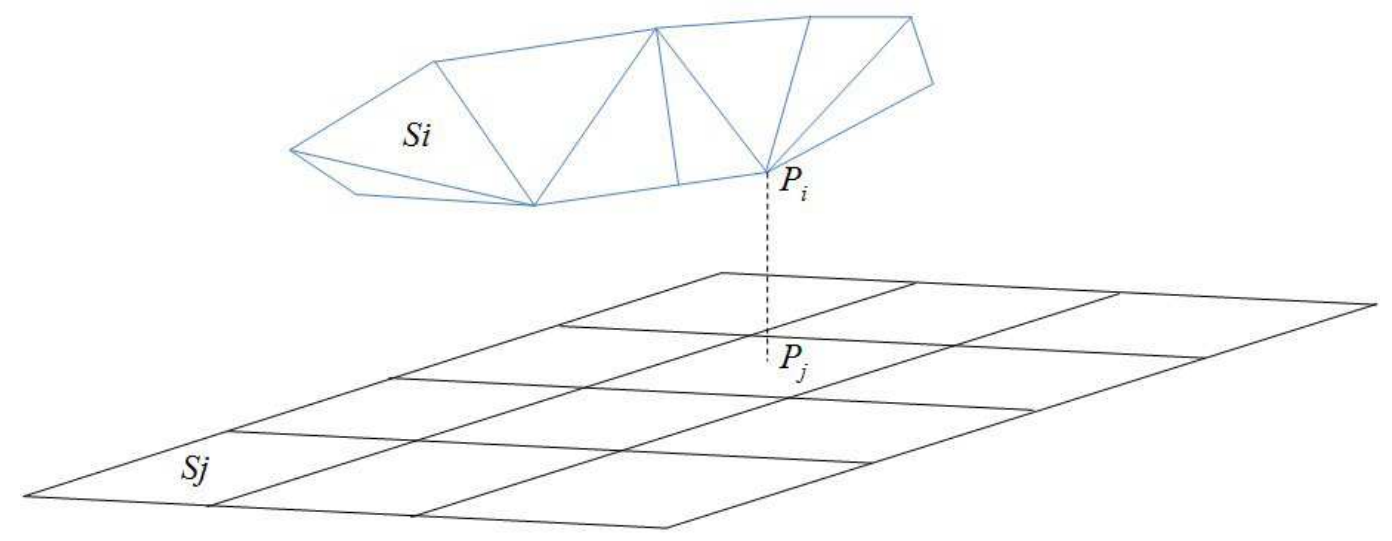

Figure 6: Orthogonal projection 
In the numerical resolution presented in [9], the parameter $k^{w}$ was considered constant. Here this is not the case and the following explicit approach is proposed:

At time step $t+h$, the local wear factor $k_{i}^{w}\left(s_{j}\right)$, of node $P_{i}$, depends on the cumulative sliding distance $s_{j}(t)$ of $P_{j}$ computed at previous time step $t$.

In order to give to the reader an overall vision of the numerical approach, the method of resolution is summarized in the following Algorithm 1. The new developments presented in this paragraph appear in italic. 
Compute mass matrix

For each cycle do

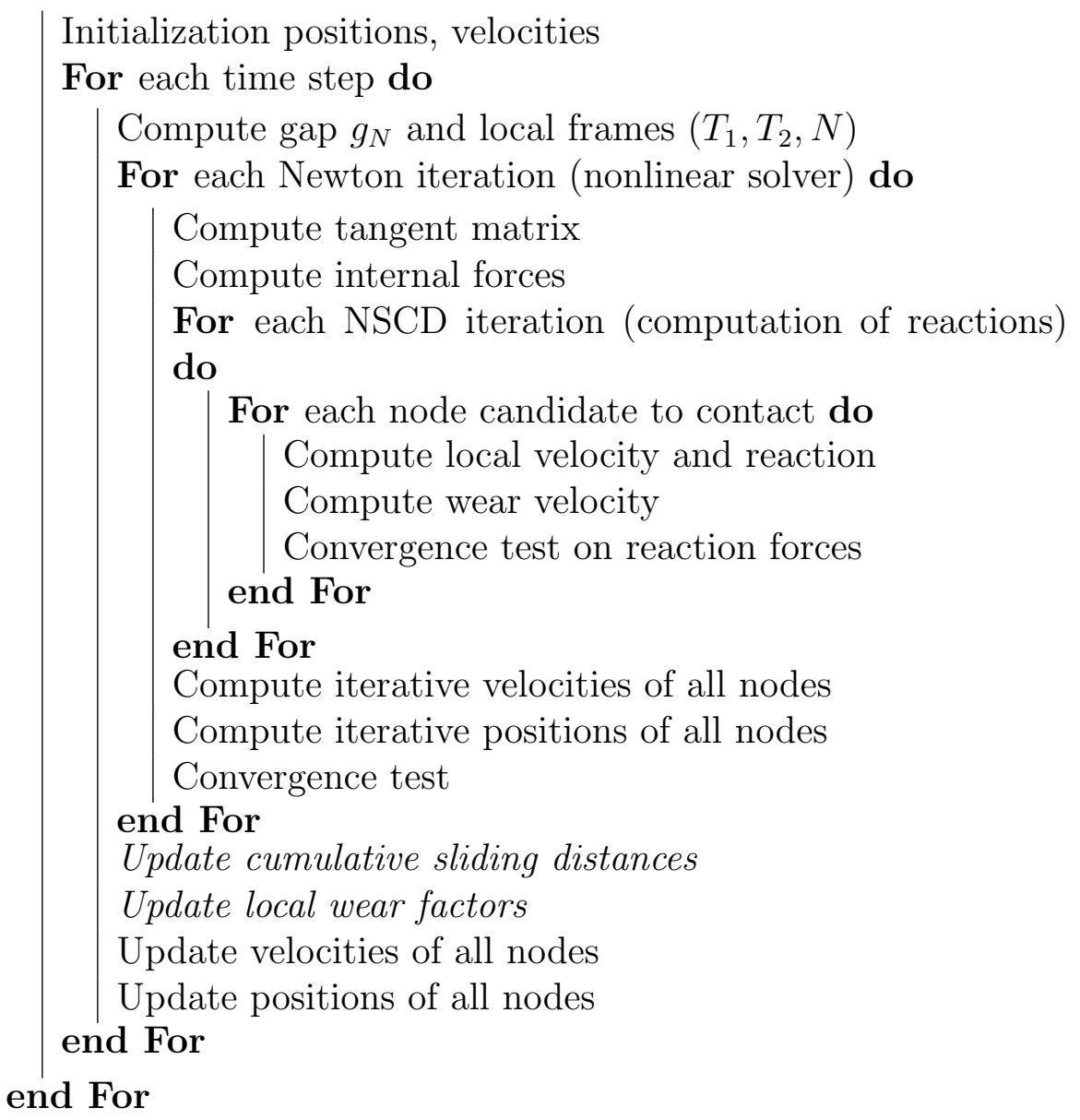

Algorithm 1: Overview of the method. Italic items represent new developements

\section{Numerical results}

\subsection{Data set}

The numerical simulations carried out in this study were built to reproduce the experimental tests proposed by Turell et al.[3]. 
The chromium-cobalt plate $S 2$ was assumed to be rigid. The upper surface of the plate of $20 \times 20 \mathrm{~mm}^{2}$ was discretized by a regular grid constituted by 40.000 quadrangular elements. Every element represents a friction zone in which each particle have a same cumulative sliding distance.

The wear factor $K_{2}$ was neglected and $K_{1}\left(s_{2}\right)=K\left(s_{2}\right)$ was given by the law of figure 7. The shape of this law was inspired by the results of Shi [4]. Wear values were fitted to be agree with experimental results obtained by Turell et al.[3].

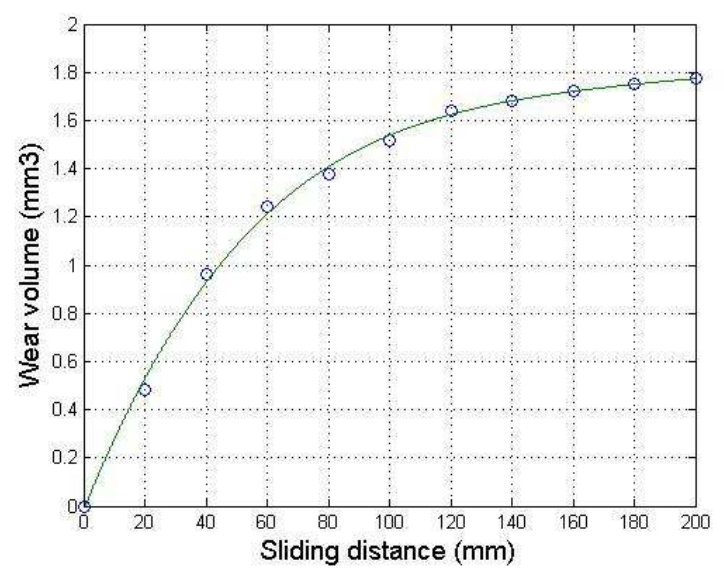

Figure 7: Wear law for computations: wear volume loss $\left(\mathrm{mm}^{3}\right)$ as a function of the sliding distance $(\mathrm{mm})$

The cylinder $S 1$ was discretized by 540 tetrahedral elements. The material characteristics of the UHMWPE were : a Young modulus of $1016 \mathrm{MPa}$, a Poisson rate of 0.46 and a mass density of $938 \mathrm{Kg} / \mathrm{m}^{3}$. The friction coefficient was 0.07 .

All wear simulation tests were conduced with a constant applied load of $200 \mathrm{~N}$.

\subsection{Results}

In the first serie of tests, as in Turell et al.[3], six motion path patterns were tested: $5 \mathrm{~mm} \times 5 \mathrm{~mm}, 4 \mathrm{~mm} \times 6 \mathrm{~mm}, 3 \mathrm{~mm} \times 7 \mathrm{~mm}, 2 \mathrm{~mm} \times 8 \mathrm{~mm}, 1 \mathrm{~mm} \times 9 \mathrm{~mm}$, $0 \mathrm{~mm} \times 10 \mathrm{~mm}$. Wear volume losses are gathered in figure 8 .

The results of simulations in figure 8 show an increase in the volume of wear according to the type of path pattern. These results were obtained by the approach developed in the preceding paragraph, with a nonlinear wear 


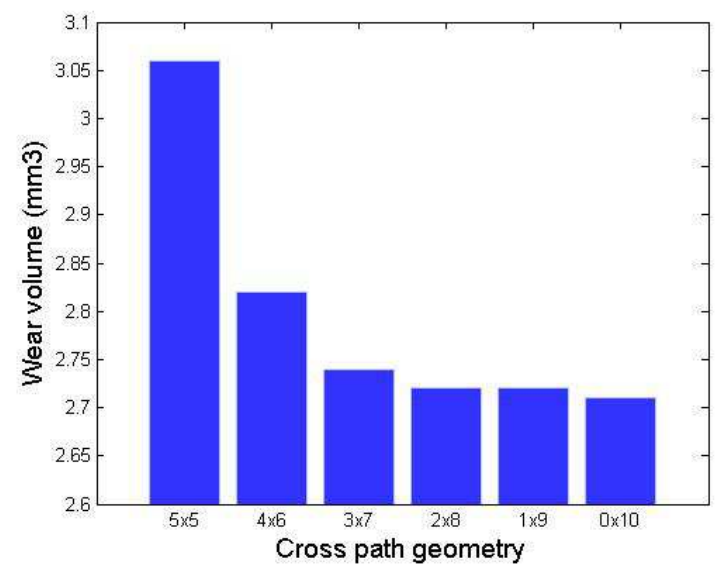

Figure 8: Wear volume loss $\left(\mathrm{mm}^{3}\right)$ versus motion path pattern $(\mathrm{mm} \times \mathrm{mm})$

rate resulting from the law of wear (figure 7). One observes approximately $13 \%$ of increase between the rectangular path pattern $5 \mathrm{mmx} 5 \mathrm{~mm}$ and the rectilinear path pattern $0 \mathrm{mmx} 10 \mathrm{~mm}$.

Figure 9 shows that the distribution of wear is relatively homogeneous at the end of the calculation. This simulation resulted from the rectangular path pattern $5 \mathrm{mmx} 5 \mathrm{~mm}$.

Further simulations with rectangular path patterns of types $6.5 \mathrm{mmx} 6 \mathrm{~mm}$, $10 \mathrm{mmx} 10 \mathrm{~mm}, 12 \mathrm{mmx} 12 \mathrm{~mm}$, preserving the same traversed total distance (just the number of cycles is changing), produced the volumes of wear represented in figure 10. The results show that volumes of wear increase according to the surface of the rectangle. The volume of wear is maximum for path pattern $12 \mathrm{mmx} 12 \mathrm{~mm}$. This behavior confirms the observations of the first series of numerical tests (figure 8). There seems to be a correlation between the surface of the rectangle and the volume of wear. To confirm this, let us continue by analyzing the friction surfaces.

Figure 11 represents the distribution of cumulative sliding distance on the plate, for a fine mesh (40.000 quadrangular elements). It is observed that the rectangular path pattern $10 \mathrm{mmx} 10 \mathrm{~mm}$ admits maximum rubbed surface, but that the cumulative sliding distance $s$ is minimum. According to the theory developed in this paper, rubbed surfaces are thus more abrasive, therefore the volume of wear is greater.

The correlation between the volume of wear and rubbed surface is illustrated in figure 12. Rubbed surface was calculated by multiplying the total 


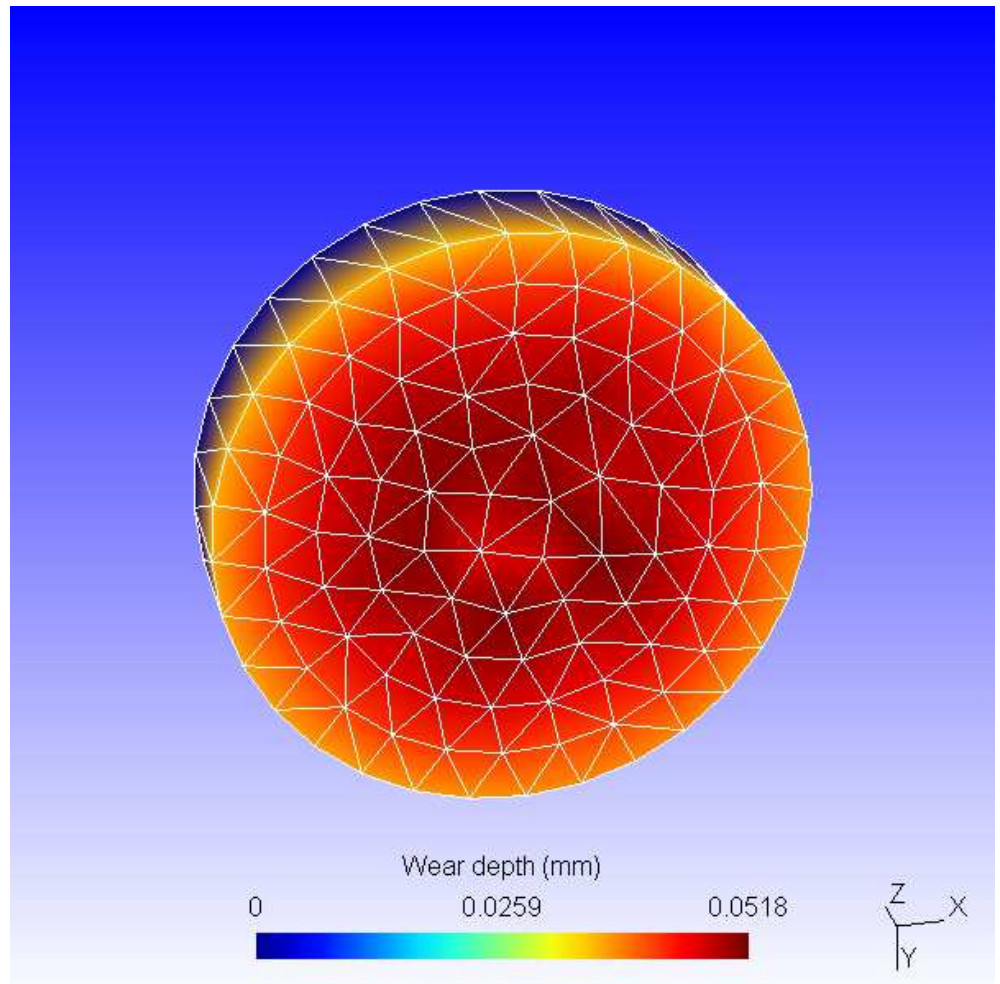

Figure 9: Wear depth on pin (mm)

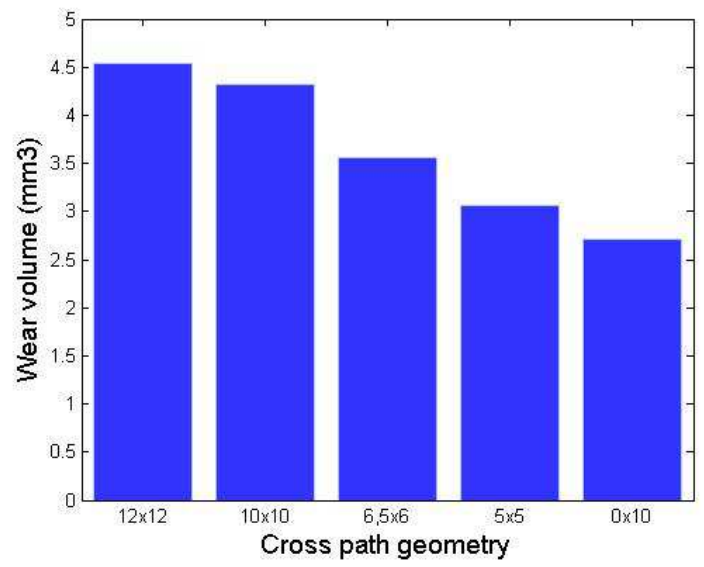

Figure 10: Wear volume loss $\left(\mathrm{mm}^{3}\right)$ versus motion path pattern $(\mathrm{mm} \times \mathrm{mm})($ serie 2$)$ 

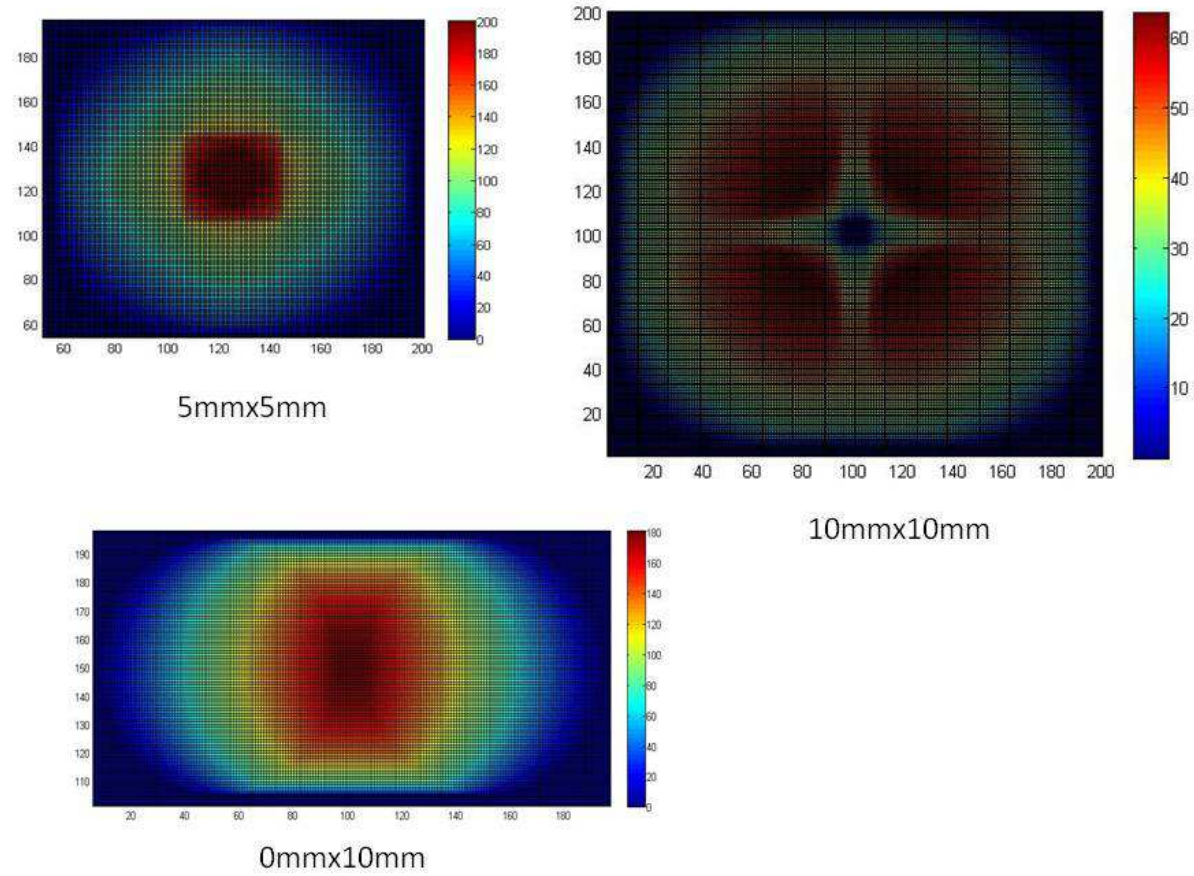

$10 \mathrm{~mm} \times 10 \mathrm{~mm}$

Figure 11: Rubbed surface and cumulative sliding distance (color map) (mm) on $S 2$

number of quadrangular elements where $s$ is nonnull by the surface of one element. This graph shows that the relation between these two parameters is not linear. The slope of the curve tends to decrease when the surface of friction increases.

\subsection{Discussion}

The numerical results show that the approach adopted in this article complies with the measurements observed by Turell et al.[3] and Laurent et al.[2]. The rectangular path patterns increase volumes of wear compared to a rectilinear path. A factor 1.7 can be observed between the minimum (0mmx10mm) and the maximum $(12 \mathrm{mmx} 12 \mathrm{~mm})$. This shows that the approach adopted in this study is relevant.

The numerical results show that there is a correlation between the entire friction surface on the plate and the volume of wear. The greater the surface the greater the volume of wear. This remains faithful to the model developed 


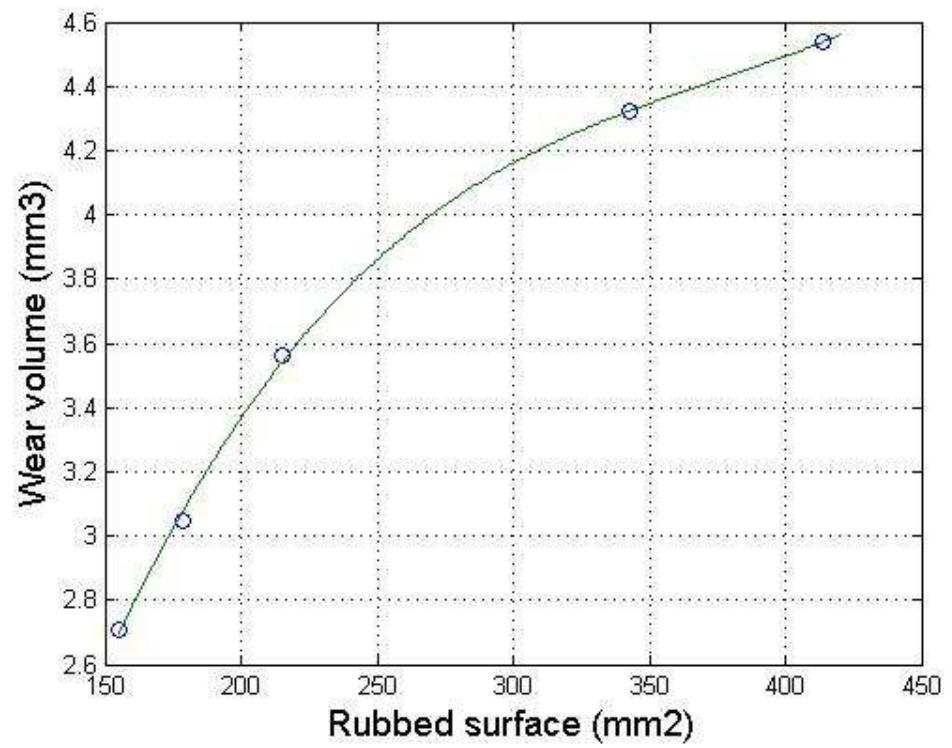

Figure 12: Wear volume versus rubbed surface

in this article. If the friction surface is wider, its abrasive capacity is stronger. However, the abrasive potential tends to decrease beyond a certain friction surface.

However the increases in wear revealed by simulations were not as spectacular as the experimental results. These numerical results were on average very far removed from Turell et al.[3]'s measurements (see figure 13). Moreover, simulations did not seem to reproduce the drop in volume of wear observed in experiments after the path pattern $3 \mathrm{mmx} 7 \mathrm{~mm}$. This decrease in wear volume was not only observed by Turell et al.[3], but also by Laurent et al.[2]. The model proposed in this paper cannot model this phenomenon.

In addition and to supplement this study, is necessary to note that for a constant wear rate, i.e. for a linear law of wear, the volume of wear calculated is of $1,8 \mathrm{~mm}^{3}$ for a rectilinear path pattern. This result is to be compared with the volume of wear $2,71 \mathrm{~mm}^{3}$ obtained by the nonlinear approach. In this case, the increase between the rectilinear path pattern with the linear approach and the rectangular path pattern $5 \mathrm{mmx} 5 \mathrm{~mm}$ with the nonlinear approach is approximately $70 \%$. It is significant and justifies the approach adopted in this article. In other words, a prediction of wear using the method adopted in [9] is computed with a relative error of $70 \%$ for a rectangular path 


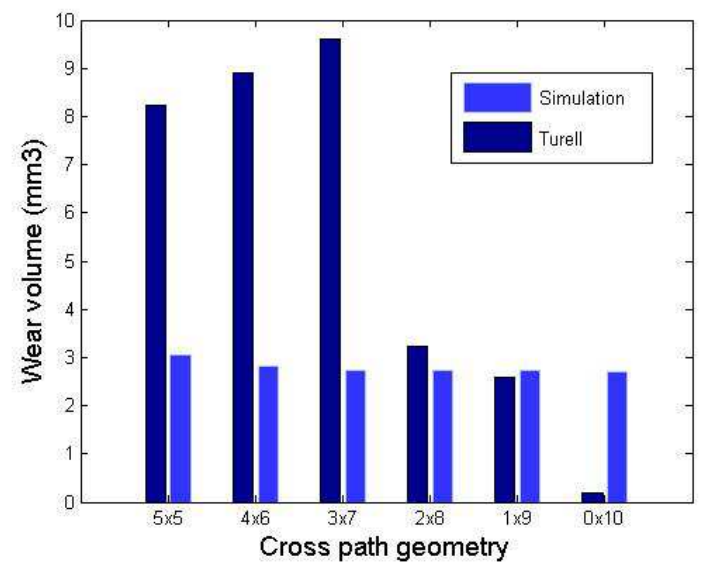

Figure 13: Wear volume loss $\left(\mathrm{mm}^{3}\right)$ versus motion path pattern $(\mathrm{mm} \times \mathrm{mm})($ comaprisons between simulations and experimentations)

pattern $5 \mathrm{mmx} 5 \mathrm{~mm}$.

\section{Conclusion}

The model of wear developed in this article is based on experimental observations collected in the literature. These highlight wear volume loss as a nonlinear function of the number of cycles. From this report a numerical wear model was developed. It was built on a local law of wear inspired by Archard's law, but with a nonconstant wear rate. This wear rate was assumed to be a function of a cumulated variable of sliding distance. Simulations show that part of the increase in multi-directional wear can be explained by the nonlinearity of wear versus the sliding distance. However, this nonlinearity does not explain all the experimental observations. It can justify the increase of wear up to factor two or even three, but not beyond. Although it is not insignificant, it is not sufficient. Different phenomena may be able to justify an increase up to a factor of 10 even 50. Some of them could be the molecular orientation of the PolyEthylene material and the orientation softening effect.

\section{References}

[1] A. Wang, A unified theory of wear for ultra-high molecular weight polyethylene in multi-directional sliding, Wear 248 (2001) 38-47. 
[2] M. P. Laurent, T. S. Johnson, J. Q. Yao, C. R. Blanchard, R. D. Crowninshield, In vitro lateral versus medial wear of a knee prosthesis, Wear 255 (2003) 1101-1106.

[3] M. Turell, A. Wang, A. Bellare, Quantication of the effect of cross-path motion on the wear rate of ultra-high molecular weight polyethylene, Wear 255, issues 7-12 (2003) 1034-1039.

[4] W. Shi, X. Y. Li, H. Dong, Improved wear resistance of ultra-high molecular weight polyethylene by plasma immersion ion implantation, Wear Volume 250, issues 1-12 (2001) Pages 544-552.

[5] M. Chandrasekaran, L. Y. Wei, K. K. Venkateshwaran, A. W. Batchelor, N. L. Loh, Tribology of uhmwpe tested against a stainless steel counterface in unidirectional sliding in presence of model synovial fluids: part 1, Wear Volume 223, Issues 1-2 (1998) Pages 13-21.

[6] S. A. R. Hashmi, S. Neogi, A. Pandey, N. Chand, Sliding wear of pp/uhmwpe blends: effect of blend composition, Wear Volume 247, Issue 1 (2001) Pages 9-14.

[7] L. Kang, A. L. Galvin, T. D. Brown, Z. Jin, F. Fisher, Quantification of the effect of cross-shear on the wear of conventional and highly cross-linked uhmwpe, Journal of Biomechanics Volume 41, Issue 2 (2008) Pages 340-346.

[8] N. Stromberg, Finite element treatment of two-dimensional thermoelastic wear problems, Comp. Meth. Appl. Mech. Engrg. 177 (1998) 441-455.

[9] F. Jourdan, A. Samida, An implicit numerical method for wear modeling applied to a hip joint prosthesis problem, Computer Methods in Applied Mechanics and Engineering Volume 198, Issues 27-29 (2009) Pages 22092217 .

[10] F. Jourdan, Numerical wear modeling in dynamics and large strains : application to knee joint prostheses, Wear 261 (2006) 283-292.

[11] P. Samyn, L. Van Schepdael, J. Leendertz, A. Gerber, W. Van Paepegem, P. De Baets, J. Degrieck, Large-scale friction 
and wear tests on a hybrid uhmwpe-pad/primer coating combination used as bearing element in an extremely high-loaded ball-joint, Tribology International Volume 39, Issue 8 (2006) Pages 796-811.

[12] J. F. Archard, Contact and rubbing of flat surfaces, J. Appl. Phys. 24 (8) (1953) 981-988.

[13] M. Jean, The non-smooth contact dynamics method, Comp. Meth. Appl. Mech. Engrg 177 (1999) 235-257.

[14] F. Jourdan, P. Alart, M. Jean, An alternative method between implicit and explicit schemes devoted to frictional contact problems in deep drawing simulation, Journal of Materials Processing Technology Volumes 80-81 (1998) 257-262.

[15] L. Adélaïde, F. Jourdan, C. Bohatier, Frictional contact solver and mesh adaptation in space-time finite element method, European Journal of Mechanics - A/Solids 22, Issue 4 (2003) 633-647.

[16] F. Jourdan, P. Alart, M. Jean, A gauss seidel like algorithm to solve frictional contact problems, Comput. Methods Appl. Mech. Engrg. 155 (1998) 31-47. 